\title{
Targeting pure quantum states by strong noncommutative dissipation
}

\author{
Vladislav Popkov, ${ }^{1,2, *}$ Carlo Presilla, ${ }^{3,4}$ and Johannes Schmidt ${ }^{5}$ \\ ${ }^{1}$ HISKP, University of Bonn, Nussallee 14-16, 53115 Bonn, Germany. \\ ${ }^{2}$ Centro Interdipartimentale per lo studio di Dinamiche Complesse, \\ Università di Firenze, via G. Sansone 1, 50019 Sesto Fiorentino, Italy \\ ${ }^{3}$ Dipartimento di Fisica, Sapienza Università di Roma, Piazzale Aldo Moro 2, Roma 00185, Italy \\ ${ }^{4}$ Istituto Nazionale di Fisica Nucleare, Sezione di Roma 1, Roma 00185, Italy \\ ${ }^{5}$ Institut für Teoretische Physik, Universität zu Köln, Zülpicher Strasse 77, Köln, Germany.
}

(Dated: November 9, 2018)

\begin{abstract}
We propose a solution to the problem of realizing a predefined and arbitrary pure quantum state, based on the simultaneous presence of coherent and dissipative dynamics, noncommuting on the target state and in the limit of strong dissipation. More precisely, we obtain a necessary and sufficient criterion whereby the nonequilibrium steady state (NESS) of an open quantum system described by a Lindblad master equation approaches a target pure state in the Zeno regime, i.e., for infinitely large dissipative coupling. We also provide an explicit formula for the characteristic dissipative strength beyond which the purity of the NESS becomes effective, thus paving the way to an experimental implementation of our criterion. For an illustration, we deal with targeting a Bell state, an arbitrary pure state of $N$ qubits, and a spin-helix state of $N$ qubits.
\end{abstract}

\section{INTRODUCTION}

Preparation of entangled states is a core problem in most protocols of quantum information science and its technological applications. Target quantum states can be generated, in principle, in various ways: via coherent dynamics, dissipative dynamics, or a generic combination of both coherent and dissipative dynamics [1-19].

The combined dissipative generation of pure states, which best mimics the typical experimental conditions, has the advantage of being stable against decoherence and almost independent of the initial conditions. Both these features are due to the existence of a nonequilibrium steady state (NESS), assumed to be unique, of the Lindblad master equation containing a non-unitary part that models a dissipative coupling of a quantum system to the environment. Apart from limitations due to imperfections of experimental setups, an ideal target might be unreachable because of fundamental limitations. An optimization problem then arises, namely, how to approach the target maximally closely with the available resources. The task can be framed in a more general context of problems of optimal control of open quantum systems [20] with vast technological applications [21].

In the present context, the ideal target is to generate a chosen pure NESS dissipatively, i.e., to obtain a pure state solution, $\rho=|\Psi\rangle\langle\Psi|$, of a stationary master equation of Lindblad form $[22,23]$

$-\frac{i}{\hbar}[H, \rho]+\sum_{\alpha} \Gamma_{\alpha}\left(L_{\alpha} \rho L_{\alpha}^{\dagger}-\frac{1}{2}\left(L_{\alpha}^{\dagger} L_{\alpha} \rho+\rho L_{\alpha}^{\dagger} L_{\alpha}\right)\right)=0$.

To accomplish this task the available resources are: the dissipative actions (the Lindblad operators $L_{\alpha}$ ), the accessible ranges of the respective dissipative strengths (the parameters $\Gamma_{\alpha}$ ), and the accessible coherent evolutions (the effective

\footnotetext{
*E-mail:popkov@uni-bonn.de
}

Hamiltonian $H$ ). It is known $[24,25]$ that an exact pure NESS of the above master equation requires a commuting action of the coherent and dissipative parts of the dynamics on the target state. More precisely, the target state $|\Psi\rangle$ must satisfy

$$
\begin{aligned}
& H|\Psi\rangle=\lambda|\Psi\rangle, \\
& L_{\alpha}|\Psi\rangle=0, \quad \text { for all } \alpha .
\end{aligned}
$$

Often, Eqs. (1) and (2) cannot be simultaneously satisfied, making the ideal target unreachable.

Suppose, however, that one can still satisfy, or approximately satisfy, some of Eqs. (1) and (2). Which conditions are crucial and which can be relaxed without ruining the NESS? A universal answer to this question does not exist, as it would require a perturbative analysis of a concrete Liouvillean operator.

Our aim is to demonstrate that one can generate an almost pure NESS by a weaker criterion than the "commutativity on the state", at the cost of increasing the strength of the dissipation. This strength can somewhat be manipulated in experiments, e.g., a dissipative loss rate of atoms in an optical trap is controlled by a laser beam intensity. Even the Zeno limit, namely, the limit of infinitely large dissipative couplings $\Gamma_{\alpha}$, can be reached experimentally [26-29], and it often produces surprising effects [30-34]. Another important aspect of our criterion is that, except for its strength, the dissipation is regarded as fixed, whereas it is $H$ to be considered adjustable, i.e., $H$ plays the role of the control parameter providing the desidered nature of the target state $|\Psi\rangle$. Again, this point of view appears to be experimentally approachable [35].

According to our weaker criterion, coherent and dissipative actions on a target state need not be commutative. Violation of the "commutativity on the state" forbids, in a strict mathematical sense, to generate an exact pure NESS. Instead, a state, which is arbitrarily close to the exact one, up to a controlled error, can be dissipatively generated. Our criterion thus naturally consists of two parts, given by following theorems 1 and 2. Theorem 1 introduces the weaker criterion valid for strong dissipation. The criterion essentially consists of a condition 


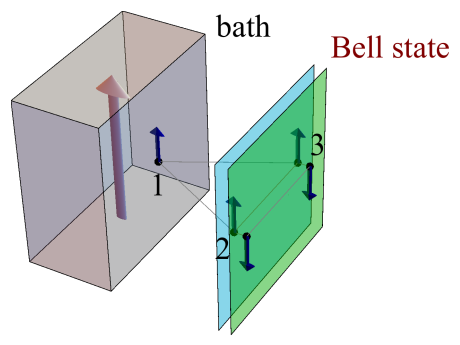

Figure 1. Targeting a Bell state $\frac{1}{\sqrt{2}}\left(\left|\uparrow_{2} \uparrow_{3}\right\rangle+\left|\downarrow_{2} \downarrow_{3}\right\rangle\right)$ in a system of $N=3$ qubits by locally coupling qubit 1 to a fully polarizing bath. Targeting criterion (5) is satisfied by the Hamiltonian $H$ defined in Fig. 3.

which relates the effective Hamiltonian $H$, the pure NESS $|\Psi\rangle$, and the dark state of the dissipator, namely, the eigenvector corresponding to zero eigenvalue of the Lindbladian in the limit of infinitely large dissipation. In formulating this criterion, we suppose that the dissipation acts only on a subset of the degrees of freedom of the system, typically a small subset, as in the case of boundary driven systems. Theorem 2 quantifies how strong the dissipation should be in order to generate an almost pure NESS, and imposes further (exceptional) restrictions on the Hamiltonian. These restrictions concern isolated points where the weak criterion alone fails or might fail. Thus, theorems 1 and 2 provide, respectively, necessary and sufficient conditions for generating a pure NESS in the Zeno limit.

To illustrate theorems 1 and 2, we discuss three examples: the generation of a Bell state of two qubits in a system of three interacting qubits (see Fig. 1), its $N$-qubit generalization, namely, the generation of an arbitrary pure state of $N$ qubits in a system of $N+1$ interacting qubits, and, finally the generation of a factorized spin-helix state in a boundary driven spin chain with nearest-neighbor interaction (see Fig. 2). Note that, as it will be clear from the precise statement of our criterion, in all these example we consider an enlarged system to obtain the effectively desired pure NESS, e.g., $N+1$ qubits for a state of $N$ qubits. In the first example, we illustrate the presence of exceptional points where the criterion may fail. In the second one, we explicitly show how a NESS approaches a pure state in the limit of large dissipative strength and evaluate the NESS relaxation time. With the third example we demonstrate how, for a given set of resources, the "commutativity on the state" condition is never fulfilled, while our weaker condition can easily be met by tuning a model parameter. In all examples, the most common type of qubit dissipation is considered, namely, the polarization, with an adjustable degree of one or two spins.

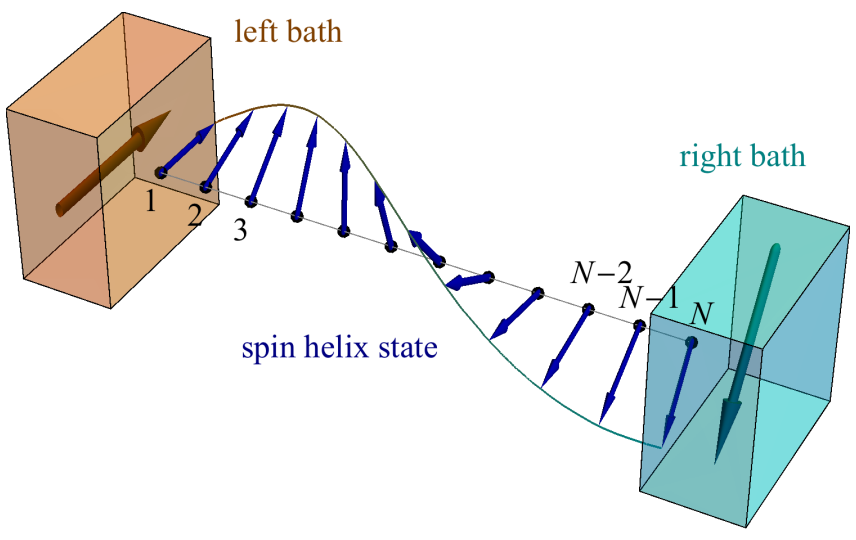

Figure 2. Targeting a spin-helix state in a chain of $N$ spins by locally coupling boundary spins 1 and $N$ to two fully polarizing baths. Targeting criterion (5) is satisfied by assuming $H$ as the Hamiltonian of the $X X Z$ Heisenberg spin $1 / 2$ model.

\section{MAIN RESULTS}

To formalize the problem, consider a finite quantum system in contact with an external environment. The time evolution of the reduced density matrix of the system $\rho$ is described by a quantum master equation in the Lindblad form [36-38] (we set $\hbar=1$ ),

$$
\frac{\partial \rho}{\partial t}=\mathcal{L} \rho=-i[H, \rho]+\Gamma \mathcal{D}_{L} \rho,
$$

where $H$ represents the unitary part of the evolution of the reduced density matrix, and $\mathcal{D}_{L}$ is the Lindblad dissipator. For simplicity, we suppose that the dissipator contains a single Lindblad operator,

$$
\mathcal{D}_{L} \rho=L \rho L^{\dagger}-\frac{1}{2}\left(L^{\dagger} L \rho+\rho L^{\dagger} L\right),
$$

with the jump operator $L$ acting in a subspace $\mathcal{H}_{0}$ of the whole Hilbert space $\mathcal{H}$ of the system. We also suppose, this in an important point, that the kernel of $\mathcal{D}_{L}$ is nondegenerate and pure in $\mathcal{H}_{0}$, i.e., it exists a unique pure state of $\mathcal{H}_{0}$, indicated by $\left|\psi_{\text {Zeno }}\right\rangle$, such that $\mathcal{D}_{L}\left|\psi_{\text {Zeno }}\right\rangle\left\langle\psi_{\text {Zeno }}\right|=0$. Note that we have $\mathcal{H}=\mathcal{H}_{0} \otimes \mathcal{H}_{1}$, with $\mathcal{H}_{0}, \mathcal{H}_{1}$ having finite dimensions $d_{0}$ and $d_{1}$, respectively. The state $\left|\psi_{\text {Zeno }}\right\rangle$ should not be confused with the desired target state, which we call $\left|\psi_{\text {target }}\right\rangle$ and which resides in $\mathcal{H}_{1}$. With an abuse of notation, which, however, should not generate troubles, the product state $|\Psi\rangle=\left|\psi_{\text {Zeno }}\right\rangle \otimes\left|\psi_{\text {target }}\right\rangle$ will also be designated as the target state in the space $\mathcal{H}$. As it will be clear from the statement of the theorems given below, we use a part of the degrees of freedom of the system, namely, $\mathcal{H}_{0}$, as an auxiliary subspace to achieve, in the Zeno limit, the desired target state in $\mathcal{H}_{1}$. This constitutes our minimal setup. Note that the reduced density matrix $\rho$ which appears in Eqs. (3) and (4) belongs to the full Hilbert space $\mathcal{H}$. Finally, we assume that the NESS, i.e., the time-independent solution of Eq. (3), that we denote as $\rho_{\text {NESS }}(\Gamma)$, is unique for any $\Gamma$. The necessary 
and sufficient criterion for the generation of a pure NESS $|\Psi\rangle$ in the Zeno limit are given by the following theorems 1 and 2 .

Theorem 1 (necessary condition) If $\lim _{\Gamma \rightarrow \infty} \rho_{\mathrm{NESS}}(\Gamma)=$ $|\Psi\rangle\langle\Psi|$, with $|\Psi\rangle=\left|\psi_{\text {Zeno }}\right\rangle \otimes\left|\psi_{\text {target }}\right\rangle$ and $\left|\psi_{\text {target }}\right\rangle \in \mathcal{H}_{1}$, then the Hamiltonian H satisfies

$$
H|\Psi\rangle=\lambda|\Psi\rangle+\kappa\left|\psi_{\text {Zeno }}^{\perp}\right\rangle \otimes\left|\psi_{\text {target }}\right\rangle,
$$

where $\left\langle\psi_{\text {Zeno }} \mid \psi_{\text {Zeno }}^{\perp}\right\rangle=0$.

The proof of theorem 1 is given in Appendix A. Note that Eq. (5) entails the noncommutativity of the coherent and dissipative parts of the dynamics on the state $|\Psi\rangle$, namely, $H L|\Psi\rangle \neq L H|\Psi\rangle$. Indeed, $H L|\Psi\rangle=0$, since $L|\Psi\rangle=0$ by the Zeno regime assumption. On the other hand, $L H|\Psi\rangle=$ $\kappa L\left|\psi_{\text {Zeno }}^{\perp}\right\rangle \otimes\left|\psi_{\text {target }}\right\rangle \neq 0$, for any nonzero $\kappa$.

Theorem 2 provides a sufficient condition for $\rho_{\text {NESS }}$ to converge to a pure target state in the Zeno limit and shows that this state is approached algebraically, as $1-\operatorname{tr} \rho_{\mathrm{NESS}}^{2}(\Gamma)=$ $\left(\Gamma_{\mathrm{ch}} / \Gamma\right)^{2}+O\left(\Gamma^{-3}\right)$, everywhere, except at isolated points where criterion (5) may break down. The measure $\epsilon(\Gamma)=$ $1-\operatorname{tr} \rho_{\mathrm{NESS}}^{2}(\Gamma)$ is chosen as a convenient measure of purity as it is strictly positive for a mixed state and vanishes if and only if $\rho_{\text {NESS }}$ is pure. Theorem 2 also gives an explicit expression for the characteristic dissipative strength $\Gamma_{\mathrm{ch}}$ beyond which an almost pure NESS can be reached.

To enunciate our result, we need to introduce some convenient notations. Let the vectors $\left|e^{j}\right\rangle, j=0,1, \ldots, d_{0}-1$ form an orthonormal basis in $\mathcal{H}_{0}$. Without loss of generality, we choose $\left|e^{0}\right\rangle \equiv\left|\psi_{\text {Zeno }}\right\rangle$ and $\left|e^{1}\right\rangle \equiv\left|\psi_{\text {Zeno }}^{\perp}\right\rangle$. Block-decompose $H$ in the basis of $\left|e^{j}\right\rangle$ as

$$
H=\sum_{j=0}^{d_{0}-1} \sum_{k=0}^{d_{0}-1} H_{j k}, \quad H_{j k}=\left|e^{j}\right\rangle\left\langle e^{k}\right| \otimes h_{j k},
$$

where $h_{j k}=\left\langle e^{j}|H| e^{k}\right\rangle$ is a Hamiltonian acting in $\mathcal{H}_{1}$. Let the eigenvectors of $h_{00},|\alpha\rangle$, where $h_{00}|\alpha\rangle=\lambda_{\alpha}|\alpha\rangle$, form an orthonormal basis in $\mathcal{H}_{1}$. Note that $h_{j k}^{\dagger}=h_{k j}$ due to the Hermiticity of $H$.

Theorem 2 (sufficient condition) Let the Hamiltonian $H$ satisfy the condition (5) with $\left|\psi_{\text {target }}\right\rangle \equiv|0\rangle, \lambda \equiv \lambda_{0}$, and $\kappa \neq$ 0 . Then, $\lim _{\Gamma \rightarrow \infty} \rho_{\text {NESS }}(\Gamma)=|\Psi\rangle\langle\Psi|$, with $|\Psi\rangle=\left|\psi_{\text {Zeno }}\right\rangle \otimes$ $\left|\psi_{\text {target }}\right\rangle$. Moreover, $\lim _{\Gamma \rightarrow \infty} \Gamma^{2}\left(1-\operatorname{tr} \rho_{\mathrm{NESS}}^{2}(\Gamma)\right)=\Gamma_{\mathrm{ch}}^{2}$, with

$$
\Gamma_{\mathrm{ch}}^{2}=8|\kappa|^{2} \sum_{\alpha=1}^{d_{1}-1} \sum_{\beta=1}^{d_{1}-1}\left(K^{-1}\right)_{\alpha \beta} R_{\beta} .
$$

The characteristic dissipative strength $\Gamma_{\mathrm{ch}}$ is expressed in terms of the inverse of the $\left(d_{1}-1\right) \times\left(d_{1}-1\right)$ matrix $K$ with elements $K_{\alpha \beta}, \alpha, \beta=1,2, \ldots, d_{1}-1$, given by

$$
K_{\alpha \beta}=\sum_{k=1}^{d_{0}-1}\left(\left|\left\langle\alpha\left|h_{k 0}\right| \beta\right\rangle\right|^{2}-\delta_{\alpha, \beta}\left\langle\alpha\left|h_{k 0}^{\dagger} h_{k 0}\right| \alpha\right\rangle\right),
$$

and the real numbers $R_{\alpha}=\langle\alpha|F| 0\rangle\left\langle 0\left|F^{\dagger}\right| \alpha\right\rangle, \quad \alpha=$ $1,2, \ldots, d_{1}-1$, where $F=\sum_{k=1}^{d_{0}-1}\left(h_{k 1}+\left[\Lambda h_{01}, h_{k 0}\right]\right)$, and

$$
\Lambda=\sum_{\alpha=1}^{d_{1}-1} \frac{1}{\lambda_{\alpha}-\lambda_{0}}|\alpha\rangle\langle\alpha| .
$$

Criterion (5) and Eqs. (7)-(9) are our main results. Actually, for a chosen $\left|\psi_{\text {target }}\right\rangle$, they allow one to find a system (to construct a Hamiltonian $H$ ) which, once coupled to a dissipator of the form (4) via the Lindblad Eq. (3) with a dissipative strength $\Gamma \gg \Gamma_{\text {ch }}$, admits the target state $|\Psi\rangle=$ $\left|\psi_{\text {Zeno }}\right\rangle \otimes\left|\psi_{\text {target }}\right\rangle$ as NESS. Note that theorem 2 does not just provide the characteristic dissipative strength, but imposes further restrictions on $H$ by implicitly requiring a nondivergent $\Gamma_{\text {ch }}$, see below. For this reason, theorems 1 and 2 can be viewed as a rough criterion (theorem 1) and refined criterion (theorem 2) for a pure NESS generation.

Two lemmas given in the Appendix A provide necessary the technical background for the proof of the theorems. Lemma 1 shows that the Lindblad operator $L$ is not arbitrary, but is actually fixed, up to a similarity transformation, by the assumption that $\mathcal{D}_{L}$ has a nondegenerate and pure kernel in $\mathcal{H}_{0}$. The dissipator (4) is then completely fixed. In lemma 2 , we calculate the dissipator inverse $\mathcal{D}_{L}^{-1}$, that appears in the recurrence relation (A5). Lemma 2 also gives a condition for the existence of the dissipator inverse, leading to the secular conditions (A6).

The main idea behind the demonstration of the theorems is relatively simple. We start assuming that there exists an expansion of the NESS in powers of $1 / \Gamma$,

$$
\rho_{\mathrm{NESS}}(\Gamma)=\sum_{m=0}^{\infty} \frac{\rho^{(m)}}{\Gamma^{m}},
$$

convergent for sufficiently large $\Gamma$, let us say $1 / \Gamma<1 / \Gamma_{\mathrm{cr}}$. In general, we are not able to evaluate the critical dissipative strength $\Gamma_{\mathrm{cr}}$ (not to be confused with $\Gamma_{\mathrm{ch}}$ ), but we always suppose it to be inside the convergence disk of the series. Plugging the expansion (10) into the Lindblad equation (3) and comparing order by order, we get a series of conditions for the existence of the terms $\rho^{(m)}$. The conditions for the existence of $\rho^{(1)}$ provide Eq. (5). In theorem 2, viceversa, the hypothesis that $H$ satisfies the criterion (5) together with the conditions for the existence of $\rho^{(m)}$ at any order $m$ permits one to conclude that $\lim _{\Gamma \rightarrow \infty} \rho_{\text {NESS }}(\Gamma)=\left|\psi_{\text {Zeno }}\right\rangle\left\langle\psi_{\text {Zeno }}|\otimes| \psi_{\text {target }}\right\rangle\left\langle\psi_{\text {target }}\right|$ and also to evaluate $\lim _{\Gamma \rightarrow \infty} \Gamma^{2}\left(1-\operatorname{tr} \rho_{\mathrm{NESS}}^{2}(\Gamma)\right)=\Gamma_{\mathrm{ch}}^{2}$.

The characteristic dissipative strength $\Gamma_{\mathrm{ch}}$ may diverge at two different kinds of singularities: those associated with the poles of $\Lambda$ in Eq. (9) and those arising from the noninvertibility points of the matrix $K$ defined by Eq. (8). Both kinds of singularities signal an inconsistency of the $1 / \Gamma$ power expansion converging to the pure NESS in the Zeno limit. At the singular points, the NESS in the Zeno limit generically is not pure. It may also happen that $\Gamma_{\mathrm{ch}}=0$. In this case, the speed of convergence to zero of the purity $\epsilon(\Gamma)$ must be evaluated at the next $1 / \Gamma$ order.

The singularities connected with the poles of $\Lambda$ are the most interesting (see Fig. 3). They appear when the eigenvalue $\lambda_{0}$ 


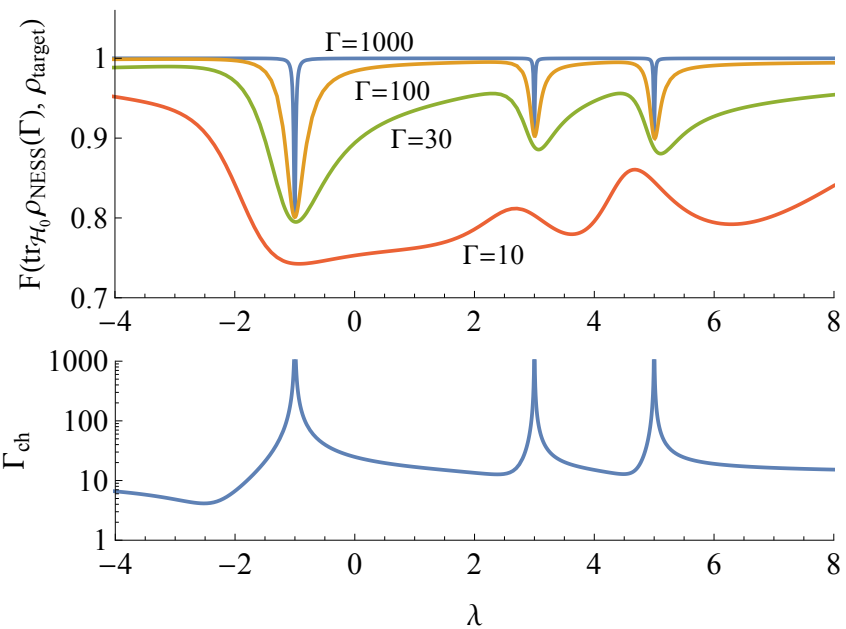

Figure 3. System of $N=3$ qubits with Lindblad operator $L=\sigma_{1}^{\dagger}$ and target state $\left|\psi_{\text {Bell }}\right\rangle=\frac{1}{\sqrt{2}}\left(\left|\uparrow_{2} \uparrow_{3}\right\rangle+\left|\downarrow_{2} \downarrow_{3}\right\rangle\right)$. Bottom panel: characteristic dissipative strength $\Gamma_{\mathrm{ch}}$ vs $\lambda$. Top panel: fidelity $F\left(\operatorname{tr}_{\mathcal{H}_{0}} \rho_{\mathrm{NESS}}(\Gamma), \rho_{\text {target }}\right)$ vs $\lambda$ for $\Gamma=10,30,100,1000$. For $\Gamma \gg \Gamma_{\mathrm{ch}}$, the NESS approaches the pure target state $\left|\uparrow_{1}\right\rangle \otimes$ $\left|\psi_{\text {Bell }}\right\rangle$ for all $\lambda$ except the degeneracy points $\lambda=\lambda_{\alpha}, \alpha=$ $1,2,3$. The Hamiltonian $H$ of the model is defined by specifying the matrix elements of the blocks $h_{00}, h_{01}, h_{10}, h_{11}$ in the orthonormal basis $\{|\alpha\rangle\}$, where $|0\rangle=\left|\psi_{\text {Bell }}\right\rangle,|1\rangle=\left|\uparrow_{2} \downarrow_{3}\right\rangle$, $|2\rangle=\left|\downarrow_{2} \uparrow_{3}\right\rangle,|3\rangle=\frac{1}{\sqrt{2}}\left(\left|\uparrow_{2} \uparrow_{3}\right\rangle-\left|\downarrow_{2} \downarrow_{3}\right\rangle\right)$. We set $\left\langle\alpha\left|h_{00}\right| \beta\right\rangle=$ $\lambda_{\alpha} \delta_{\alpha, \beta}$, with $\lambda_{0} \equiv \lambda, \lambda_{1}=-1, \lambda_{2}=3$ and $\lambda_{3}=5$, $\left\langle 0\left|h_{10}\right| 0\right\rangle=0.7$ and $\left\langle\alpha\left|h_{10}\right| 0\right\rangle=0$, with $\alpha=1,2,3$. All the other matrix elements are assigned according to the following formula: $\left\langle\alpha\left|h_{01}\right| \beta\right\rangle=Q_{\alpha+1, \beta+5}+$ H.c., $\left\langle\alpha\left|h_{10}\right| \beta\right\rangle=$ $Q_{\alpha+5, \beta+1}+$ H.c., $\left\langle\alpha\left|h_{11}\right| \beta\right\rangle=\frac{1}{2}\left(Q_{\alpha+5, \beta+5}+\right.$ H.c. $)$, where $Q_{k, j}=i^{k-j}\left(\bmod \left(\left\lfloor 7 \tan \left(k^{7} j^{4}\right)\right\rfloor, 2\right)+0.7\right)$. Note that for each value of the parameter $\lambda$, we have a different Hamiltonian $H(\lambda)$.

of $h_{00}$, corresponding to the eigenstate $|0\rangle$, becomes degenerate. There can be at most $d_{1}-1$ singularity points $\lambda_{0}=\lambda_{\beta}$, $\beta=1, \ldots d_{1}-1$. Occasionally, by vanishing of the matrix element $\left\langle\alpha\left|\left[\Lambda h_{01}, h_{k 0}\right]\right| 0\right\rangle$, the $\alpha$-th singularity can be removed.

On the other hand, singularities due to non-invertibility of the matrix $K$ are rare since they occur only by simultaneous vanishing of several entries of the Hamiltonian (see Appendix A). We demonstrate both kinds of singularities in the following example.

\section{TWO-QUBIT BELL STATE}

As the simplest nontrivial example, still interesting from an applicative point of view, we target a Bell state of two qubits. As shown in Fig. 1, to realize this state we consider a system of $N=3$ qubits and a Lindblad operator acting on the first qubit only, $L=\sigma^{+} \otimes I \otimes I$. Note that a Bell state of two qubits cannot be a dark state of the operators $\sigma^{+} \otimes I$ or $I \otimes \sigma^{+}$. Therefore, for a dissipative generation of a Bell state of two qubits in the Zeno regime at least three qubits are needed. The degrees of freedom of one qubit, that
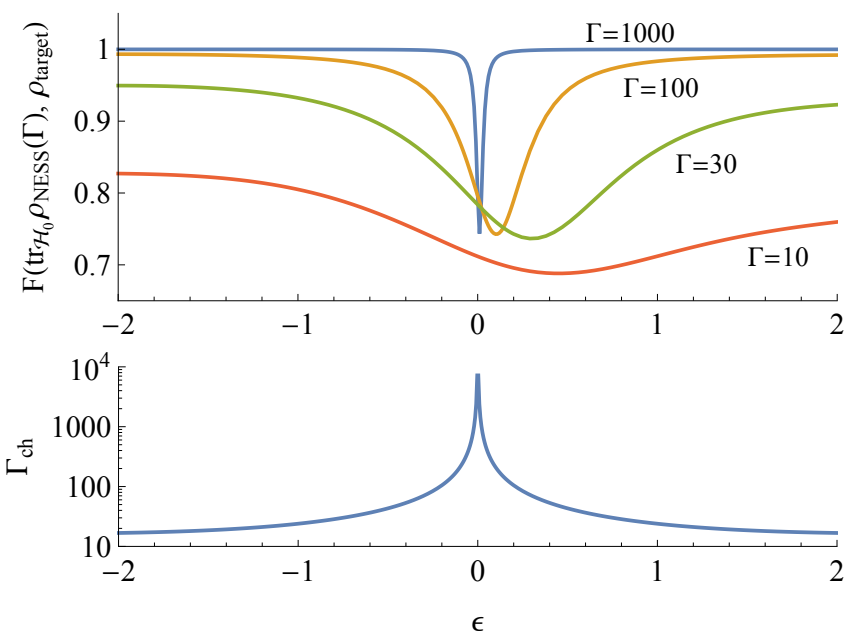

Figure 4. As in Fig. 3, but with the characteristic dissipative strength and the fidelity plotted as a function of the Hamiltonian parameter $\epsilon=\left\langle 0\left|h_{10}\right| 1\right\rangle$. We have also set $\left\langle 0\left|h_{10}\right| \alpha\right\rangle=0$, for $\alpha=2,3$, while the rest of the Hamiltonian matrix elements are equal to those of Fig. 3 with $\lambda=1$. For $\Gamma \gg \Gamma_{\text {ch }}$, the NESS approaches the pure target state $\left|\uparrow_{1}\right\rangle \otimes\left|\psi_{\text {Bell }}\right\rangle$ for all $\epsilon$ except the singular point $\epsilon=0$, where the matrix $K$ of Eq. (8) is not invertible.

we designate as qubit 1 , play the role of the auxiliary subspace $\mathcal{H}_{0}$. In the Hilbert subspace $\mathcal{H}_{0} \equiv \mathbb{C}_{2}$, we choose the standard basis vectors $\left\{\left|e^{0}\right\rangle,\left|e^{1}\right\rangle\right\}=\{|\uparrow\rangle,|\downarrow\rangle\}$. Note that $\left|e^{0}\right\rangle \equiv\left|\psi_{\text {Zeno }}\right\rangle$ is the unique pure state of $\mathcal{H}_{0}$ such that $\mathcal{D}_{L}\left|\psi_{\text {Zeno }}\right\rangle\left\langle\psi_{\text {Zeno }}\right|=0$. We also have, necessarily, in view of the dimension $d_{0}=2,\left|e^{1}\right\rangle \equiv\left|\psi_{\text {Zeno }}^{\perp}\right\rangle$. In the complement Hilbert subspace $\mathcal{H}_{1} \equiv \mathbb{C}_{2} \otimes \mathbb{C}_{2}$, the target state reads

$$
\left|\psi_{\text {Bell }}\right\rangle=\frac{1}{\sqrt{2}}\left(\left|\uparrow_{2} \uparrow_{3}\right\rangle+\left|\downarrow_{2} \downarrow_{3}\right\rangle\right),
$$

where subscripts denote the embedding. To make sure that the Lindblad Eq. (3) admits, in the Zeno limit, the pure NESS $|\uparrow\rangle\left\langle\uparrow|\otimes| \psi_{\text {Bell }}\right\rangle\left\langle\psi_{\text {Bell }}\right|$, we just have to find a Hamiltonian $H$ that satisfies the criterion (5) with $\left|\psi_{\text {Bell }}\right\rangle \equiv|0\rangle, \lambda \equiv \lambda_{0}$, and $\kappa \neq 0$. Specifically, this amounts to determining the matrix elements of the blocks $h_{00}, h_{01}, h_{10}, h_{11}$ of $H$ in the orthonormal basis $\{|\alpha\rangle\}_{\alpha=0}^{3}$, constituted by the eigenvectors of $h_{00}, h_{00}|\alpha\rangle=\lambda_{a}|\alpha\rangle$, with the constraint $|0\rangle=\left|\psi_{\text {Bell }}\right\rangle$. For simplicity, let us, first, choose the basis of $\mathcal{H}_{1}$ given by $|0\rangle=\left|\psi_{\text {Bell }}\right\rangle,|1\rangle=\left|\uparrow_{2} \downarrow_{3}\right\rangle,|2\rangle=\left|\downarrow_{2} \uparrow_{3}\right\rangle$, and $|3\rangle=$ $\frac{1}{\sqrt{2}}\left(\left|\uparrow_{2} \uparrow_{3}\right\rangle-\left|\downarrow_{2} \downarrow_{3}\right\rangle\right)$, and then define $h_{00}$ in this basis as the diagonal matrix $h_{00}=\operatorname{diag}\left(\lambda_{0}, \lambda_{1}, \lambda_{2}, \lambda_{3}\right)$. The criterion (5) just imposes the following other conditions: $\left\langle 0\left|h_{10}\right| 0\right\rangle=\kappa$ and $\left\langle\alpha\left|h_{10}\right| 0\right\rangle=0, \alpha=1,2,3$. For the rest, besides being Hermitian, the Hamiltonian $H$ can be chosen in an arbitrary way, as long as the NESS remains unique.

In Fig. 3, with $H$ chosen as specified in the caption, we show, as a function of the parameter $\lambda \equiv \lambda_{0}$, the behavior of the fidelity between the target state $\rho_{\text {target }}=\left|\psi_{\text {Bell }}\right\rangle\left\langle\psi_{\text {Bell }}\right|$ and the stationary solution of Eq. (3) traced in $\mathcal{H}_{0}$, with $\rho_{\text {NESS }}(\Gamma)$ evaluated numerically for different values of $\Gamma$. In the bottom panel, for the same values of $\lambda$, we display the 
characteristic dissipative strength $\Gamma_{\mathrm{ch}}$ calculated according to Eq. (7). It is evident that, whenever $\Gamma \gg \Gamma_{\text {ch }}$, the fidelity is close to 1 , i.e., $\operatorname{tr}_{\mathcal{H}_{0}} \rho_{\mathrm{NESS}}(\Gamma)$ approaches the pure target state. This is true for any $\lambda$ except the degeneracy points $\lambda=\lambda_{\alpha}, \alpha=1,2,3$, where the matrix $\Lambda$ of Eq. (9) is singular and $\Gamma_{\text {ch }}$ diverges. At these points, $\lim _{\Gamma \rightarrow \infty} \rho_{\text {NESS }}(\Gamma)$ exists but is not a pure state; therefore the fidelity remains different from 1 for arbitrarily large $\Gamma$.

Consider the Hamiltonian $H$ obtained from that of Fig. 3 with $\lambda=1$, by zeroing all the elements $\left\langle 0\left|h_{10}\right| \alpha\right\rangle=0$, for $\alpha>1$, and putting $\left\langle 0\left|h_{10}\right| 1\right\rangle=\epsilon$. If $\epsilon=0$, the matrix $K$ evaluated as prescribed by Eq. (8) is a stochastic matrix and $\operatorname{det} K=0$ as a consequence of the Perron-Frobenius theorem. For $\epsilon \neq 0$, $\operatorname{det} K \neq 0$ and $K$ is invertible. Then, according to Eq. (7), we expect a singularity at the point $\epsilon=0$, where $K^{-1}$ does not exist. This is exactly the singular behavior which emerges for large $\Gamma$ in Fig. 4 at $\epsilon=0$.

\section{ARBITRARY N-QUBIT PURE STATE}

It is possible to generalize the "Bell state" example provided for a system with $2+1$ qubits, to an arbitrary target pure state in a system of $N+1$ qubits. Dissipation is made to act only on one qubit, say qubit 1 , which defines an auxiliary space and allows the reaching of the target state for the remaining $N$ qubits. A minimal Hamiltonian realizing this scenario is given below. For this minimal Hamiltonian, we are able to calculate the NESS analytically for arbitrary dissipative strengths $\Gamma$.

Let us denote by $\left|e^{0} 0\right\rangle \equiv\left|e^{0}\right\rangle \otimes|0\rangle$ a pure state to be targeted in the Zeno limit. Notations are as above, namely, $\left|e^{0}\right\rangle=\left|\psi_{\text {Zeno }}\right\rangle$ and $|0\rangle=\left|\psi_{\text {target }}\right\rangle$. The evolution of the density matrix is described by Eq. (3) with one local Lindblad operator $L=\sigma_{1}^{+}$acting on the auxiliary spin 1 only.
The Lindblad operator $L$ projects the first spin onto the state $\left|e^{0}\right\rangle \equiv|\uparrow\rangle$, while $\left|e^{1}\right\rangle \equiv|\downarrow\rangle$ is the vector completing the basis of $\mathcal{H}_{0}=\mathbb{C}_{2}$. The state $|0\rangle$ is an arbitrary vector in $\mathcal{H}_{1}=\left(\mathbb{C}_{2}\right)^{\otimes_{N}}$, the Hilbert space of the remaining $N$ spins. form

A simple Hamiltonian satisfying Eqs. (5) and (A40) has the

$$
\begin{aligned}
H= & \sum_{\alpha=0}^{2^{N}-1}\left(\lambda_{\alpha}\left|e^{0} \alpha\right\rangle\left\langle e^{0} \alpha\left|+d_{\alpha}\right| e^{1} \alpha\right\rangle\left\langle e^{1} \alpha\right|\right) \\
& +\left(\kappa\left|e^{0} 0\right\rangle\left\langle e^{1} 0\right|+\text { H.c. }\right) \\
& +\sum_{\alpha=0}^{2^{N}-2}\left(\eta_{\alpha}\left|e^{0}(\alpha+1)\right\rangle\left\langle e^{1} \alpha\right|+\text { H.c. }\right) \\
& +\sum_{\alpha=1}^{2^{N}-2} \sum_{\beta>\alpha}^{2^{N}-2}\left(d_{\alpha \beta}\left|e^{1} \alpha\right\rangle\left\langle e^{1} \beta\right|+\text { H.c. }\right)
\end{aligned}
$$

where H.c. denotes the Hermitian conjugate. The terms proportional to $\lambda_{0}$ and $\kappa$ guarantee condition (5) while the terms proportional to $\eta_{\alpha}$ lift up the degeneracy of the Liouvillean. (Both $\kappa$ and all $\eta_{\alpha}$ are required to be nonzero.) All the other parameters are free parameters. The pure state $\left|e^{0} 0\right\rangle$ is exactly realized as NESS only in the Zeno limit.

It can be checked that the recurrence conditions (A5) for the Hamiltonian (12) simplify to

$$
\rho^{(m+2)}=-\frac{4 \zeta}{\left(\lambda_{0}-\lambda_{1}\right)^{2}} \rho^{(m)},
$$

where

$$
\rho^{(1)}=2 \mathrm{i} \kappa\left(\frac{\eta_{0}^{*}}{\lambda_{0}-\lambda_{1}}\left|e^{0} 0\right\rangle\left\langle e^{0} 1|+| e^{0} 0\right\rangle\left\langle e^{1} 0\right|\right)+\text { H.c. },
$$

$$
\begin{gathered}
\rho^{(2)}=4|\kappa|^{2}\left(-\frac{|\kappa|^{2}+\left|\eta_{0}\right|^{2}+\left(\lambda_{0}-\lambda_{1}\right)^{2}}{\left(\lambda_{0}-\lambda_{1}\right)^{2}}\left|e^{0} 0\right\rangle\left\langle e^{0} 0\left|+\frac{|\kappa|^{2}+\left|\eta_{0}\right|^{2}}{\left(\lambda_{0}-\lambda_{1}\right)^{2}}\right| e^{0} 1\right\rangle\left\langle e^{0} 1|+| e^{1} 0\right\rangle\left\langle e^{1} 0\right|\right) \\
+4 \kappa\left(\eta_{0}^{*} \frac{\lambda_{0}^{2}-|\kappa|^{2}-\left|\eta_{0}\right|^{2}-\lambda_{0} \lambda_{1}-d_{0}\left(\lambda_{0}-\lambda_{1}\right)}{\left(\lambda_{0}-\lambda_{1}\right)^{2}}\left|e^{0} 0\right\rangle\left\langle e^{0} 1\left|+\frac{\kappa^{*} \eta_{0}}{\lambda_{0}-\lambda_{1}}\right| e^{0} 1\right\rangle\left\langle e^{1} 0\right|\right. \\
\left.+\frac{\lambda_{0}^{2}-\left|\eta_{0}\right|^{2}-\lambda_{0} \lambda_{1}-d_{0}\left(\lambda_{0}-\lambda_{1}\right)}{\lambda_{0}-\lambda_{1}}\left|e^{0} 0\right\rangle\left\langle e^{1} 0\right|+\text { H.c. }\right)
\end{gathered}
$$

and

As a result, the NESS can be written as

$$
\begin{aligned}
\zeta= & \left(\lambda_{0}^{2}-\lambda_{0} \lambda_{1}-\left|\eta_{0}\right|^{2}-d_{0}\left(\lambda_{0}-\lambda_{1}\right)\right)^{2} \\
& +|\kappa|^{2}\left(|\kappa|^{2}+2\left(\left(\lambda_{0}-\lambda_{1}\right)^{2}+\left|\eta_{0}\right|^{2}\right)\right)
\end{aligned}
$$

$$
\begin{aligned}
\rho_{\mathrm{NESS}}= & \left|e^{0} 0\right\rangle\left\langle e^{0} 0\right| \\
& +\frac{1}{\Gamma^{2}} \sum_{n=0}^{\infty}\left(\frac{-4 \zeta}{\left(\lambda_{0}-\lambda_{1}\right)^{2} \Gamma^{2}}\right)^{n}\left(\Gamma \rho^{(1)}+\rho^{(2)}\right) .
\end{aligned}
$$




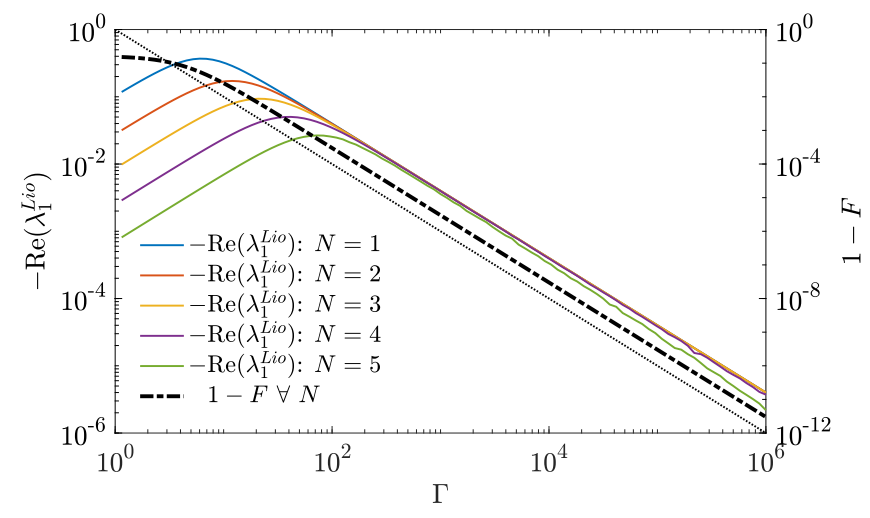

Figure 5. Complement to 1 of the fidelity of the NESS (dot-dashed bold line, right vertical axis) and minus the real part of the Liouvillian gap versus $\Gamma$ for $N=1,2,3,4,5$ (solid lines from top to bottom, left vertical axis). Note that $1-F$ is independent of $N$, and so become, for large $\Gamma$, the quantities $-\operatorname{Re}\left(\lambda_{1}^{\text {Lio }}\right)$. Parameters: $\lambda_{\alpha}=$ $1+\alpha+\sqrt{\alpha}, \kappa=1, \eta_{\alpha}=1 \forall \alpha, d_{\alpha}=0 \forall \alpha, d_{\alpha \beta}=0$. The tiny dotted line, given by $y=1 / x$ for the left $y$ axis, and by $y=1 / x^{2}$ for the right $y$ axis, is a guide for the eyes.

Summing up the geometrical series, we obtain

$$
\rho_{\mathrm{NESS}}=\left|e^{0} 0\right\rangle\left\langle e^{0} 0\right|+\frac{\left(\lambda_{0}-\lambda_{1}\right)^{2}}{4 \zeta+\left(\lambda_{0}-\lambda_{1}\right)^{2} \Gamma^{2}}\left(\Gamma \rho^{(1)}+\rho^{(2)}\right) .
$$

Formally, the Taylor expansion (17) converges for $\Gamma>\Gamma_{\mathrm{cr}}=$ $2 \sqrt{\zeta} /\left|\lambda_{0}-\lambda_{1}\right|$. However, the analytical formula (18) has no singularities and is valid for arbitrary $\Gamma$. Note that the NESS does not depend on terms proportional to $d_{\alpha}$ for $\alpha \geq 1$ and $d_{\alpha \beta}$.

The purity measure $1-\operatorname{tr} \rho_{\mathrm{NESS}}^{2}(\Gamma)$ of the NESS for finite dissipative strength is easily calculated. The Taylor expansion for large $\Gamma$ of formula (18) gives

$$
1-\operatorname{tr}\left(\rho_{\mathrm{NESS}}^{2}\right)=\frac{8|\kappa|^{4}}{\left(\lambda_{0}-\lambda_{1}\right)^{2} \Gamma^{2}}+\mathcal{O}\left(\Gamma^{-3}\right) .
$$

From this expression, we read off the characteristic dissipative strength

$$
\Gamma_{\mathrm{ch}}=\frac{\sqrt{8}|\kappa|^{2}}{\left|\lambda_{0}-\lambda_{1}\right|} .
$$

This result can also be obtained directly by using Eq. (7). Note that Eq. (20) displays a divergence for $\lambda_{1}=\lambda_{0}$, but not for other points of degeneracy of $h_{00}$, i.e., $\lambda_{\alpha}=\lambda_{0}, \alpha>1$. This happens because the matrix elements $\left\langle\alpha\left|h_{01}\right| 0\right\rangle$ vanish for all $\alpha>1$, so that system of Eqs. (A21) leads to a divergence only for $\alpha=1$ for which $\left\langle\alpha\left|h_{01}\right| 0\right\rangle \neq 0$.

Finally, the fidelity of the NESS with respect to the ideal target state $\left|e^{0} 0\right\rangle$, namely, $F=\sqrt{\left\langle e^{0} 0\left|\rho_{\text {NESS }}\right| e^{0} 0\right\rangle}$, can be explicitly calculated from Eq. (18) to be

$$
F=\sqrt{1-\frac{4|\kappa|^{2}\left(\left(\lambda_{0}-\lambda_{1}\right)^{2}+|\kappa|^{2}+\left|\eta_{0}\right|^{2}\right)}{4 \zeta+\left(\lambda_{0}-\lambda_{1}\right)^{2} \Gamma^{2}}},
$$

while the Taylor expansion of $1-F$ for large $\Gamma$ is given by

$$
1-F=\frac{2|\kappa|^{2}\left(\left(\lambda_{0}-\lambda_{1}\right)^{2}+|\kappa|^{2}+\left|\eta_{0}\right|^{2}\right)}{\left(\lambda_{0}-\lambda_{1}\right)^{2} \Gamma^{2}}+\mathcal{O}\left(\Gamma^{-4}\right)
$$

Figure 5 shows the fidelity $F$ of the NESS, actually, its complement to 1 , and the inverse of the system relaxation time $\tau_{\text {relax }}$, namely, minus the real part of the Liouvillian gap, as a function of $\Gamma$ for different (small) system sizes. We observe that $1-F \sim \Gamma^{-2}$ and $\tau_{\text {relax }}^{-1} \sim \Gamma^{-1}$, at least asymptotically in $\Gamma$. We conclude that the inverse relaxation time grows sublinearly with $1-F$, i.e.,

$$
\tau_{\text {relax }} \sim(1-F)^{-\frac{1}{2}},
$$

for $1-F$ small enough. Remarkably, both the asymptotic fidelity and the asymptotic relaxation time do not depend on the system size $N$.

\section{FACTORIZED SPIN-HELIX STATE OF $N$ QUBITS}

Our third example concerns targeting a factorized spinhelix pure state in a chain of $N$ qubits (spins 1/2), which, in the absence of dissipation, are supposed to evolve according to the paradigmatic Heisenberg Hamiltonian. The spin-helix state in a chain of $N$ qubits has the form

$$
|\Psi\rangle=\bigotimes_{k=1}^{N}\left(\begin{array}{c}
\cos \left(\frac{\theta}{2}\right) e^{-i \varphi_{k} / 2} \\
\sin \left(\frac{\theta}{2}\right) e^{i \varphi_{k} / 2}
\end{array}\right), \quad \varphi_{k}=\gamma(k-1)
$$

This state describes a precession of the local spin along the chain with fixed orbital angle $\theta$ and polar angle $\varphi$ homogeneously increasing as $\varphi_{k+1}-\varphi_{k}=\gamma$. The boundary conditions $\theta_{1}=\theta, \varphi_{1}=0$ and $\theta_{N}=\theta, \varphi_{N}=\gamma(N-1)=\phi$ can be realized by coupling the first and last spins of the chain to two fully polarizing baths. In other words, we can consider a Lindblad master equation like (3) with two dissipators, $\mathcal{D}_{L_{L}}$ and $\mathcal{D}_{L_{R}}$, of the form (4), associated with the left and right Lindblad operators, $L_{L}$ and $L_{R}$, acting only on spin 1 and $\operatorname{spin} N$, respectively. See Fig. 2 for an example with $\theta=\pi / 2$. More details on the setting of the model and its properties in various limits can be found in [39-43].

A possible protocol leading to the evolution (3) with local Lindblad operators $\mathcal{D}_{L_{L}}, \mathcal{D}_{L_{R}}$ can be found in the Appendix A of [44]. Despite the introduction of a second dissipator, for any chain of $N>3$ spins with first-neighbor interaction, $L_{L}$ and $L_{R}$ operate independently. It can be shown that the conditions for the existence of the terms $\rho^{(m)}$ in the expansion (10) have exactly the same form of Eq. (A6) in Appendix A, provided that now we intend $\mathcal{H}_{0} \equiv \mathbb{C}_{2} \otimes \mathbb{C}_{2}$. We conclude that our theorems 1 and 2 apply unchanged. We find that the criterion (5) for the spin-helix state (24) with $\lambda=0$ and $\kappa=-i \sqrt{2} \sin \theta \sin \gamma$ is satisfied by the celebrated $X X Z$ 
Heisenberg Hamiltonian,

$$
H_{X X Z}=\sum_{j=1}^{N-1}\left(\sigma_{j}^{x} \sigma_{j+1}^{x}+\sigma_{j}^{y} \sigma_{j+1}^{y}+\cos \gamma \sigma_{j}^{z} \sigma_{j+1}^{z}\right)
$$

Note that the $Z$-axis anisotropy is tuned to $\Delta=\cos \gamma$. For $\theta=\pi / 2$, convergence to a spin-helix state in the Zeno limit was suggested in [45] via a different method. The factorized state (24) is a rather unusual NESS for a quantum many-body driven system. From a quantum transport viewpoint, it carries a ballistic, system-size independent magnetization current. It is characterized via a macroscopic winding number, which counts the number of full rotations of the local magnetization vector along the chain. Moreover, since the actions of the Lindblad operators and of the Hamiltonian on the state (24) do not commute, the spin-helix state cannot be obtained within the strong criterion of Eqs. (1) and (2). On the other hand, by our weaker criterion the spin-helix state can be spotted and approached with arbitrary fidelity for sufficiently large dissipation. Further elaborate analysis shows that for any angle $\gamma$ which is a rational of $\pi, \gamma / \pi=n / m$, with integer $n, m$, convergence to a spin-helix state breaks down for systems of size $N \geq m+1$. This rather surprisingly behavior is an involved prediction of present theorem 2. Details can be found in [46]. Note that, even though it generically takes longer for a system with local Liouvillean to relax to the NESS, the typical relaxation time is expected to grow only polynomially with the system size, see [47].

Similarly to [48], the spin-helix state (24) can be used to dissipatively generate an arbitrary pure single-spin state on a remote location, arbitrarily far from the place where the dissipation acts. An experimental verification of our results should be within the reach [49] of modern experimental technics, which allow to build and probe, with single-site resolution, arrays of magnetic atoms on a surface by low-temperature scanning tunneling microscopy [35].

\section{CONCLUSIONS}

To conclude, we have obtained a general criterion for a nonequilibrium steady state to become a pure state of the form $\left|\psi_{\text {Zeno }}\right\rangle \otimes\left|\psi_{\text {target }}\right\rangle$ in the limit of strong dissipation, and calculated the relevant characteristic dissipation strength. The relative merit of our approach is the absence of any strict requirements on the Lindblad operators, besides the demand for locally targeting some pure state $\left|\psi_{\text {Zeno }}\right\rangle$. This is easily achieved by most commonly implemented dissipative mechanisms like pumping, local dissipative loss or particles from a trap. The key condition to obtain a desired $\left|\psi_{\text {target }}\right\rangle$ is then the restriction (5) imposed on the effective Hamiltonian. This becomes a sufficient criterion provided the exceptions stated by theorem 2 are circumvented.

\section{ACKNOWLEDGMENTS}

VP thanks the DFG for financial support. VP thanks G. Schütz and C. Kollath for useful discussions. We thank G. Jona-Lasinio for a critical reading.

\section{Appendix A: Proofs of theorems 1 and 2}

We start with two lemmas, which are key for the following demonstrations.

lemma 1 Let $\mathcal{D}_{L}$ be a Lindblad dissipator of the form $\mathcal{D}_{L} \rho=$ $L \rho L^{\dagger}-\frac{1}{2}\left(L^{\dagger} L \rho+\rho L^{\dagger} L\right)$ with $\rho$ density matrix operator in $\mathcal{H}$ and $L$ acting in the $d_{0}$-dimensional subspace $\mathcal{H}_{0}$ of the Hilbert space $\mathcal{H}$. If $\mathcal{D}_{L}$ has a nondegenerate pure kernel, i.e., there exists a unique pure state $|\phi\rangle$ such that $\mathcal{D}_{L}|\phi\rangle\langle\phi|=0$, then $L$ can be brought into a single Jordan block acting in $\mathcal{H}_{0}$, namely, $L=J_{0, d_{0}}$, where $\left\langle e^{i}\left|J_{0, d_{0}}\right| e^{j}\right\rangle=\delta_{i, j-1}, i, j=$ $0,1, \ldots, d_{0}-1$.

Proof of lemma 1. From the condition $\mathcal{D}_{L}|\phi\rangle\langle\phi|=0$, it follows that $L|\phi\rangle=b|\phi\rangle$. Without loss of generality, we can put $b=0$, i.e., $|\phi\rangle$ can always be made a dark state of $L$, by repartitioning the coherent and dissipative parts, $H$ and $L$, in the Lindblad master equation $\partial \rho / \partial t=-i[H, \rho]+\Gamma \mathcal{D}_{L} \rho$, see $[24,25]$. Because of the nondegeneracy assumption, no other eigenstates of $L$ exist different from $|\phi\rangle$. As a consequence, $L$ must be nondiagonalizable, meaning it is splitted into Jordan blocks by a similarity transformation, $V L V^{-1}=\tilde{L}$. Each Jordan block separately would give rise to a state $|\tilde{\phi}\rangle$ such that $\tilde{L}|\tilde{\phi}\rangle=0$, which would contradict the nondegeneracy assumption. We conclude that there must be one Jordan block only. This is exactly the definition of $J_{0, d_{0}}$, up to an arbitrary scalar factor, $\tilde{L}=a J_{0, d_{0}}$. Without loss of generality, we set $L=J_{0, d_{0}}$, since the Hamiltonian is not defined yet and the coefficient $a$ is absorbed into the dissipative strength $\Gamma$.

lemma 2 Let $\mathcal{D}_{L}$ be a Lindblad dissipator of the form specified in lemma 1 and $X$ an operator acting in $\mathcal{H}$. A necessary condition for $\mathcal{D}_{L}^{-1} X$ to exist is $\operatorname{tr}_{\mathcal{H}_{0}} X=0$, where $\operatorname{tr}_{\mathcal{H}_{0}}$ denotes the partial trace in $\mathcal{H}_{0}$. If $L=J_{0, d_{0}}$, this condition is also sufficient.

Proof of lemma 2. Suppose that $\mathcal{D}_{L}^{-1} X=Y$ exists. Then $X=\mathcal{D}_{L} Y$ and, using the cyclic invariance of the trace, we have $\operatorname{tr}_{\mathcal{H}_{0}} X=\operatorname{tr}_{\mathcal{H}_{0}} \mathcal{D}_{L} Y=0$. Assume, now, that $L=$ $J_{0, d_{0}}$. Let us define a representation of a spin $s=\left(d_{0}-1\right) / 2$ in the Hilbert space $\mathcal{H}_{0}$ of dimension $d_{0}$ and consider the basis $|n\rangle, n=0,1, \ldots, 2 s$, such that $J_{0, d_{0}}|n\rangle=|n-1\rangle$, $J_{0, d_{0}}|0\rangle=0$. Then, the dissipator $\mathcal{D}_{L}$ acts in the space spanned by $|n\rangle\langle m|$ as 


$$
\mathcal{D}_{L}|n\rangle\langle m|= \begin{cases}|n-1\rangle\langle m-1|-| n\rangle\langle m|, & n>0, m>0, \\ -\frac{1}{2}|n\rangle\langle 0|, & n>0, m=0, \\ -\frac{1}{2}|0\rangle\langle m|, & m>0, n=0, \\ 0, & n=m=0 .\end{cases}
$$

From Eq. (A1), it follows that for any matrix $X$ with zero trace, $\operatorname{tr} X=0$, we can construct $\mathcal{D}_{L}^{-1} X$. Indeed, since the dissipator $\mathcal{D}_{L}$ is a linear map, it is enough to show that every nondiagonal element of $X$ is invertible, and so is also the diagonal of $X$. An arbitrary nondiagonal element $|n\rangle\langle m|, n \neq m$, is readily inverted using Eq. (A1) as

$$
\mathcal{D}_{L}^{-1}|n\rangle\langle m|= \begin{cases}\sum_{k=0}^{n-1}|n-k\rangle\left\langle m-k\left|-\frac{1}{2}\right| 0\right\rangle\langle m-n|, & n<m, \\ \sum_{k=0}^{m-1}|n-k\rangle\left\langle m-k\left|-\frac{1}{2}\right| n-m\right\rangle\langle 0|, & n>m .\end{cases}
$$

To invert $\operatorname{diag}(X)$, we introduce diagonal matrices $\phi(k)_{i j}=\delta_{i j} \theta(k-i), i, j, k=1,2, \ldots, 2 s$, where $\theta(n)$ is a discrete Heaviside step function. Then,

$$
\mathcal{D}_{L}^{-1} \operatorname{diag}(X)=\sum_{k=1}^{2 s} \operatorname{tr}(\phi(k) \operatorname{diag}(X))(|k-1\rangle\langle k-1|-| 0\rangle\langle 0|) .
$$

We conclude that $\operatorname{tr} X=0$ is a sufficient condition to construct $\mathcal{D}_{L}^{-1} X$.

Proof of theorem 1. We start by assuming that there exists an expansion of the NESS in powers of $1 / \Gamma$,

$$
\rho_{\mathrm{NESS}}(\Gamma)=\sum_{m=0}^{\infty} \frac{\rho^{(m)}}{\Gamma^{m}}
$$

convergent for sufficiently large $\Gamma$, i.e., $1 / \Gamma<1 / \Gamma_{\mathrm{cr}}$. We will always suppose to be inside the convergence disk.

According to the hypothesis and the choice made for the basis in $\mathcal{H}_{0}$, we have as $\rho^{(0)}=\left|e^{0}\right\rangle\left\langle e^{0}|\otimes| \psi_{\text {target }}\right\rangle\left\langle\psi_{\text {target }}\right|$ with $\left|e^{0}\right\rangle \equiv\left|\psi_{\text {Zeno }}\right\rangle$. Substituting the expansion (A4) into the Lindblad master equation, and comparing order by order, we obtain: $\mathcal{D}_{L} \rho^{(0)}=0$ and

$$
\rho^{(m+1)}=i \mathcal{D}_{L}^{-1}\left[H, \rho^{(m)}\right]+M^{(m+1)},
$$

where $M^{(m+1)} \in \operatorname{ker} \mathcal{D}_{L}$. Note that due to the Hermiticity of $\rho_{\text {NESS }}$, and the property $\operatorname{tr} \rho_{\text {NESS }}=1$, it follows $\left(M^{(m)}\right)^{\dagger}=$ $M^{(m)}$ and $\operatorname{tr} M^{(m)}=0$ for all $m$. For the expansion (A4) to be consistent, the existence of an operator inverse in (A5) is required at any order $m>0$. Lemma 1 allows us to assume $L=J_{0, d_{0}}$ so that, according to lemma 2 , the existence of the inverse $\mathcal{D}_{L}^{-1}$ at the $m$-th order is granted by

$$
\operatorname{tr}_{\mathcal{H}_{0}}\left[H, \rho^{(m)}\right]=0, \quad m=0,1, \ldots,
$$

where $\operatorname{tr}_{\mathcal{H}_{0}}$ denotes the partial trace in Hilbert space $\mathcal{H}_{0}$. In addition, we require a nontriviality condition, which guarantees that the NESS becomes a pure state only in the Zeno limit, whereas it is a mixed state for any finite $\Gamma$. Such a condition can be written as

$$
\left[H, \rho^{(0)}\right] \neq 0,
$$

and amounts to have $\kappa \neq 0$ in Eq. (5).
The existence of the expansion (A4) implies that the consistency conditions (A6) are to be satisfied at any order. Using the decomposition $H=\sum_{j=0}^{d_{0}-1} \sum_{k=0}^{d_{0}-1} H_{j k}$, with $H_{j k}=$ $\left|e^{j}\right\rangle\left\langle e^{k}\right| \otimes h_{j k}$, we have

$$
\begin{aligned}
{\left[H, \rho^{(0)}\right] } & =\left[H,\left|e^{0}\right\rangle\left\langle e^{0}|\otimes| \psi_{\text {target }}\right\rangle\left\langle\psi_{\text {target }}\right|\right] \\
& =\sum_{k=1}^{d_{0}-1}\left(H_{k 0} \rho^{(0)}-\rho^{(0)} H_{0 k}\right)
\end{aligned}
$$

so that the condition (A6) for $m=0$ gives

$$
\operatorname{tr}_{\mathcal{H}_{0}}\left[H, \rho^{(0)}\right]=\left[h_{00},\left|\psi_{\text {target }}\right\rangle\left\langle\psi_{\text {target }}\right|\right]=0,
$$

entailing

$$
h_{00}\left|\psi_{\text {target }}\right\rangle=\lambda\left|\psi_{\text {target }}\right\rangle .
$$

Thus $\left|\psi_{\text {target }}\right\rangle$ must be an eigenstate of $h_{00}$, which we choose to identify as the eigenstate $\alpha=0$, namely, $\left|\psi_{\text {target }}\right\rangle \equiv|0\rangle$ and $\lambda \equiv \lambda_{0}$.

At the next order of the $1 / \Gamma$ power expansion, $\rho^{(1)}$ is constructed applying $\mathcal{D}_{L}^{-1}$ to the commutator (A8). To proceed, we note that for any $k>0$ and for any $\alpha, \beta$ we have $\mathcal{D}_{L}\left|e^{0} \alpha\right\rangle\left\langle e^{k} \beta\left|=-\frac{1}{2}\right| e^{0} \alpha\right\rangle\left\langle e^{k} \beta\right|$, and $\mathcal{D}_{L}\left|e^{k} \alpha\right\rangle\left\langle e^{0} \beta\right|=$ $-\frac{1}{2}\left|e^{k} \alpha\right\rangle\left\langle e^{0} \beta\right|$, where we introduced the notation $\left|e^{j} \alpha\right\rangle=$ $\left|e^{j}\right\rangle \otimes|\alpha\rangle$. We obtain

$$
\rho^{(1)}=M^{(1)}-2 i \sum_{k=1}^{d_{0}-1}\left(H_{k 0} \rho^{(0)}-\rho^{(0)} H_{0 k}\right) .
$$

Because $M^{(1)}$ is an element of the kernel of $\mathcal{D}_{L}$, we rewrite it as $M^{(1)}=\sum_{\alpha, \beta} M_{\alpha, \beta}^{(1)}\left|e^{0} \alpha\right\rangle\left\langle e^{0} \beta\right|$, with $M_{\alpha, \beta}^{(1)}$ unknown coefficients. By plugging expression (A11) into Eq. (A6) for 
$m=1$, we get

$$
\begin{aligned}
\operatorname{tr}_{\mathcal{H}_{0}}\left[H, i \rho^{(1)}\right] & =\operatorname{tr}_{\mathcal{H}_{0}}\left(-4 \sum_{k=1}^{d_{0}-1} \mathcal{D}_{H_{k 0}} \rho^{(0)}+\left[H_{00}, M^{(1)}\right]\right) \\
& =0 .
\end{aligned}
$$

In the above expression, $\mathcal{D}_{H_{k 0}}$ is a dissipator of the form (4) with $L \rightarrow H_{k 0}$, and we used $h_{j k}^{\dagger}=h_{k j}$. Equation (A12) provides $d_{1}^{2}$ scalar equations $\left\langle\alpha\left|\operatorname{tr}_{\mathcal{H}_{0}}\left[H, i \rho^{(1)}\right]\right| \beta\right\rangle=0, \alpha, \beta=$ $0,1, \ldots, d_{1}-1$. In particular, for $\alpha=\beta=0$ we obtain, after some algebra,

$$
\left\langle 0\left|\operatorname{tr}_{\mathcal{H}_{0}}\left[H, i \rho^{(1)}\right]\right| 0\right\rangle=\sum_{k=1}^{d_{0}-1} \sum_{\gamma=1}^{d_{1}-1}\left|\left\langle 0\left|h_{0 k}\right| \gamma\right\rangle\right|^{2}=0,
$$

leading to $\left\langle\gamma\left|h_{j 0}\right| 0\right\rangle=\kappa_{j} \delta_{\gamma, 0}$ for any $j>0$. Without loss of generality, we can choose the vectors $\left|e^{j}\right\rangle$, with $j>0$, of the orthonormal basis in $\mathcal{H}_{0}$ in such a way that $\kappa_{1} \equiv \kappa \neq 0$ and $\kappa_{j}=0$ for $j>1$. In this basis, we have

$$
\left\langle\gamma\left|h_{k 0}\right| 0\right\rangle=\lambda \delta_{k, 0} \delta_{\gamma, 0}+\kappa \delta_{k, 1} \delta_{\gamma, 0},
$$

equivalent to Eq. (5) with $\left|e^{1}\right\rangle \equiv\left|\psi_{\text {Zeno }}^{\perp}\right\rangle$.

Proof of theorem 2. According to the hypothesis, and with the identifications $\left|e^{0}\right\rangle \equiv\left|\psi_{\text {Zeno }}\right\rangle,\left|e^{1}\right\rangle \equiv\left|\psi_{\text {Zeno }}^{\perp}\right\rangle$, $|0\rangle \equiv\left|\psi_{\text {target }}\right\rangle$ and $\lambda_{0} \equiv \lambda$, the Hamiltonian $H$ is such that $h_{00}|0\rangle=\lambda_{0}|0\rangle$ and $h_{10}|0\rangle=\kappa|0\rangle, \kappa \neq 0$.

If the consistency conditions (A6) were satisfied at any order $m$, the $1 / \Gamma$ power expansion (A4) would allow us to state that $\lim _{\Gamma \rightarrow \infty} \rho_{\mathrm{NESS}}(\Gamma)=\rho^{(0)}$. Supposing for a moment that this is the case, and observing that $\rho^{(0)}=\left|e^{0}\right\rangle\left\langle e^{0}|\otimes| 0\right\rangle\langle 0|$ satisfies Eq. (A10), in virtue of the unicity of the NESS, we achieve the thesis $\lim _{\Gamma \rightarrow \infty} \rho_{\mathrm{NESS}}(\Gamma)=|\Psi\rangle\langle\Psi|$, with $|\Psi\rangle=\left|\psi_{\text {Zeno }}\right\rangle \otimes\left|\psi_{\text {target }}\right\rangle$. Therefore, it remains to demonstrate that the consistency conditions (A6) are satisfied for any $m>0$.

Condition (A6) for $m=1$ is given by Eq. (A12) and is equivalent to $d_{1}{ }^{2}$ scalar equations obtained by taking the expectation of (A12) between any two states $\langle\alpha|$ and $|\beta\rangle$. The scalar equation obtained for $\alpha=\beta=0$, namely, Eq. (A13), is now satisfied by hypothesis (5). We split the remaining $d_{1}{ }^{2}-1$ equations in in three parts:

$$
\begin{aligned}
& i\left[h_{00}, m^{(1)} \Phi\right]=-2 h_{01} h_{10} \Phi+2|\kappa|^{2} \Phi, \\
& i\left[h_{00}, \Phi m^{(1)}\right]=-2 \Phi h_{01} h_{10}+2|\kappa|^{2} \Phi, \\
& {\left[h_{00}, m_{V}^{(1)}\right]=0,}
\end{aligned}
$$

where we have introduced

$$
\begin{aligned}
& m^{(1)}=\operatorname{tr}_{\mathcal{H}_{0}} M^{(1)}=\sum_{\alpha=0}^{d_{1}-1} \sum_{\beta=0}^{d_{1}-1} M_{\alpha \beta}^{(1)}|\alpha\rangle\langle\beta|, \\
& m_{V}^{(1)}=\sum_{\alpha=1}^{d_{1}-1} \sum_{\beta=1}^{d_{1}-1} M_{\alpha \beta}^{(1)}|\alpha\rangle\langle\beta|, \\
& \Phi=\operatorname{tr}_{\mathcal{H}_{0}} \rho^{(0)}=|0\rangle\langle 0| .
\end{aligned}
$$

Equation (A15) is a closed set of linear equations for the $d_{1}-1$ unknowns $M_{\alpha 0}^{(1)}, \alpha>0$, which, using (A14), can be written as

$$
\sum_{\gamma=1}^{d_{1}-1}\left(\left\langle\alpha\left|h_{00}\right| \gamma\right\rangle-\lambda_{0} \delta_{\alpha, \gamma}\right) M_{\gamma 0}^{(1)}=2 i \kappa\left\langle\alpha\left|h_{01}\right| 0\right\rangle .
$$

The unknowns $M_{\alpha 0}^{(1)}$ are uniquely determined from (A21) if $\operatorname{det}\left\|\left\langle\alpha\left|h_{00}\right| \gamma\right\rangle-\lambda_{0} \delta_{\alpha, \gamma}\right\| \neq 0$, or, equivalently, if the eigenvalue $\lambda_{0}$ of the $d_{1} \times d_{1}$ matrix $h_{00}$ is nondegenerate. In the basis where $h_{00}$ is diagonal, $\left\langle\alpha\left|h_{00}\right| \gamma\right\rangle=\delta_{\alpha, \gamma} \lambda_{\alpha}$, so we obtain $M_{\alpha 0}^{(1)}=2 i \kappa\left\langle\alpha\left|h_{01}\right| 0\right\rangle /\left(\lambda_{\alpha}-\lambda_{0}\right)$, or, in matrix form,

$$
m^{(1)} \Phi-M_{00}^{(1)} \Phi=2 i \Lambda h_{01} h_{10} \Phi,
$$

where $\Lambda$ is given by Eq. (9).

Equation (A16) is the Hermitian conjugate of Eq. (A15) and leads to $M_{0 \alpha}^{(1)}=\overline{M_{\alpha 0}^{(1)}}$.

Equation (A17) is a set of $\left(d_{1}-1\right)^{2}$ equations that do not determine $m_{V}^{(1)}$ completely but restrict it to have common eigenvectors with $h_{00}$. If the spectrum of $h_{00}$ is nondegenerate, then $m_{V}^{(1)}$ is automatically diagonal in the basis $|\alpha\rangle$; otherwise, it can be made diagonal,

$$
\begin{aligned}
& m_{V}^{(1)}=\sum_{\alpha=1}^{d_{1}-1} \mu_{\alpha}|\alpha\rangle\langle\alpha|, \\
& h_{00}=\sum_{\alpha=0}^{d_{1}-1} \lambda_{\alpha}|\alpha\rangle\langle\alpha|,
\end{aligned}
$$

where all $\lambda_{\alpha}, \mu_{\alpha}$ are real. To determine $\mu_{\alpha}$, additional relations are needed which come from Eq. (A6) for $m=2$. Calculating $\rho^{(2)}=i \mathcal{D}_{L}^{-1}\left[H, \rho^{(1)}\right]+M^{(2)}$, with $M^{(2)}=$ $\sum_{\alpha \beta} M_{\alpha \beta}^{(2)}\left|e^{0} \alpha\right\rangle\left\langle e^{0} \beta\right|$, we obtain

$$
\begin{aligned}
\rho^{(2)}= & M^{(2)}-2 i\left[H, M^{(1)}\right]+8 \mathcal{D}_{H} \rho^{(0)} \\
& +4\left(\kappa^{2} \rho^{(0)}-H_{10} \Omega H_{01}\right) .
\end{aligned}
$$

We then split the set of $d_{1}^{2}$ equations (A6) for $m=2$ into five parts:

$$
\begin{aligned}
0= & \operatorname{tr}_{\mathcal{H}_{0}}\left[H, \rho^{(2)}\right] \\
= & \sum_{\alpha=0}^{d_{1}-1} \sum_{\beta=0}^{d_{1}-1} Z_{\alpha \beta}|\alpha\rangle\langle\beta| \\
= & Z_{00}|0\rangle\left\langle 0\left|+\sum_{\alpha=1}^{d_{1}-1} Z_{\alpha 0}\right| \alpha\right\rangle\left\langle 0\left|+\sum_{\alpha=1}^{d_{1}-1} Z_{0 \alpha}\right| 0\right\rangle\langle\alpha| \\
& +\sum_{\alpha=1}^{d_{1}-1} \sum_{\beta=1}^{d_{1}-1}\left(1-\delta_{\alpha, \beta}\right) Z_{\alpha \beta}|\alpha\rangle\left\langle\beta\left|+\sum_{\alpha=1}^{d_{1}-1} Z_{\alpha \alpha}\right| \alpha\right\rangle\langle\alpha| .
\end{aligned}
$$


First, consider the $d_{1}-1$ equations $Z_{\alpha \alpha}=0$. After some algebra, using (A23) we obtain

$$
Z_{\alpha \alpha}=4 i\left\langle\alpha\left|\sum_{k=1}^{d_{0}-1} \mathcal{D}_{h_{k 0}} m_{V}^{(1)}\right| \alpha\right\rangle=\sum_{\beta=1}^{d_{1}-1} K_{\alpha \beta} \mu_{\beta}=0 .
$$

where $K_{\alpha \beta}$ are given by (8). Now, if $\operatorname{det} K \neq 0$, then all $\mu_{\beta}=0$, and therefore $m_{V}^{(1)}=0$.

Consider now $Z_{00}=0$. Using $Z_{00} \Phi=\Phi \operatorname{tr}_{\mathcal{H}_{0}}\left[H, \rho^{(2)}\right] \Phi$, after some algebra we obtain

$$
\begin{aligned}
Z_{00} \Phi & =-2 i \Phi \operatorname{tr}_{\mathcal{H}_{0}}\left[H,\left[H, M^{(1)}\right]\right] \Phi \\
& =2 i\left(\Phi m^{(1)} h_{01} h_{10} \Phi+\Phi h_{01} h_{10} m^{(1)} \Phi\right) .
\end{aligned}
$$

Substituting $h_{01} h_{10} \Phi$ and $\Phi h_{01} h_{10}$ from Eqs. (A15) and (A16), we find $Z_{00} \Phi=4 i|\kappa|^{2} \Phi m^{(1)} \Phi=0$, so that

$$
M_{00}^{(1)}=0 \text {. }
$$

The matrix $M^{(1)}$ is thus completely determined

$$
\begin{aligned}
M^{(1)} & =\left|e^{0}\right\rangle\left\langle e^{0}\right| \otimes\left(m^{(1)} \Phi+\Phi m^{(1)}\right) \\
& =2 i\left|e^{0}\right\rangle\left\langle e^{0}\right| \otimes\left(\Lambda h_{01} h_{10} \Phi-\Phi h_{01} h_{10} \Lambda\right) .
\end{aligned}
$$

The set of equations $Z_{\alpha \beta}=0$, for $\alpha, \beta>0$ with $\alpha \neq \beta$, after some algebra is brought in the form

$$
\left\langle\alpha\left|\left[h_{00}, \tilde{m}_{V}^{(2)}\right]\right| \beta\right\rangle=0
$$

where

$$
\begin{gathered}
\tilde{m}_{V}^{(2)}=m_{V}^{(2)}-m^{(1)} \Phi m^{(1)}, \\
m^{(2)}=\operatorname{tr}_{\mathcal{H}_{0}} M^{(2)}=\sum_{\alpha=0}^{d_{1}-1} \sum_{\beta=0}^{d_{1}-1} M_{\alpha \beta}^{(2)}|\alpha\rangle\langle\beta|, \\
m_{V}^{(2)}=\sum_{\alpha=1}^{d_{1}-1} \sum_{\beta=1}^{d_{1}-1} M_{\alpha \beta}^{(2)}|\alpha\rangle\langle\beta|,
\end{gathered}
$$

and it entails the diagonal nature of the matrix $\tilde{m}_{V}^{(2)}$ in the eigenbasis of $h_{00}$,

$$
\tilde{m}_{V}^{(2)}=\sum_{\alpha=1}^{d_{1}-1} q_{\alpha}|\alpha\rangle\langle\alpha|
$$

The equations $Z_{\alpha 0}=0$ and $Z_{0 \alpha}=0, \alpha>0$, determine the first column and the first row of the matrix $M^{(2)}$, except for the element $M_{00}^{(2)}$. Using Eqs. (A14), (A15) and (A16), these equations can be written in the form

$$
\left[h_{00}, m^{(2)}-2 \lambda_{0} m^{(1)}\right] \Phi=Q \Phi-\Phi Q \Phi
$$

where

$$
Q=-4 i \sum_{k=1}^{d_{0}-1} \mathcal{D}_{h_{k 0}} m^{(1)} \Phi-8|\kappa|^{2} h_{11} \Phi+4 \kappa \sum_{k=1}^{d_{0}-1} h_{0 k} h_{k 1} \Phi
$$

and the Hermitian conjugate equation for $\Phi m^{(2)}$. The linear problem (A36) is readily solvable as

$$
\left(m^{(2)}-2 \lambda_{0} m^{(1)}\right) \Phi-M_{00}^{(2)} \Phi=\Lambda Q \Phi .
$$

To completely determine the matrix $M^{(2)}=\left|e^{0}\right\rangle\left\langle e^{0}\right| \otimes$ $m^{(2)}$, with

$$
m^{(2)}=\Phi m^{(2)}+m^{(2)} \Phi+m_{V}^{(2)}-M_{00}^{(2)} \Phi
$$

we need the diagonal entries of $m_{V}^{(2)}$, which, according to Eq. (A35), are given by $M_{\alpha \alpha}^{(2)}=q_{\alpha}+M_{\alpha 0}^{(1)} M_{0 \alpha}^{(1)}, \alpha>0$. To find $q_{\alpha}$ it is necessary to go to the third order of the expansion and write down the set of equations $\left\langle\alpha\left|\operatorname{tr}_{\mathcal{H}_{0}}\left[H, \rho^{(3)}\right]\right| \alpha\right\rangle=0$. After quite tedious but straightforward calculations, using Eq. (A36), we obtain a closed set of equations for $q_{\beta}$ of the form

$$
\sum_{\beta=0}^{d_{1}-1} K_{\alpha \beta} q_{\beta}+\left\langle\alpha\left|\mathcal{F} \Phi \mathcal{F}^{\dagger}\right| \alpha\right\rangle=0
$$

where

$$
\mathcal{F}=\sum_{k=1}^{d_{0}-1}\left(2 i h_{k 1} h_{10}+\left[m^{(1)}, h_{k 0}\right]\right)
$$

and $K_{\alpha \beta}$ is given by Eq. (8). The solution of the linear problem (A39) exists if $\operatorname{det} K \neq 0$. Finally, $M_{00}^{(2)}$ is found from the requirement $\operatorname{tr} M^{(2)}=0$. At this point, the first two orders $\rho^{(1)}$ and $\rho^{(2)}$ of the NESS expansion (A4) are completely determined from Eqs. (A11), (A25), (A30) and (A38).

We remark that all systems of equations obtained until now, arising in the first three orders of the NESS expansion in powers of $1 / \Gamma$, Eqs. (A15), (A16), (A36) and Eqs. (A27), (A39), are governed by two $\left(d_{1}-1\right) \times\left(d_{1}-1\right)$ matrices: the matrix $\tilde{h}_{00}$ with elements $\left(h_{00}\right)_{\alpha \beta}-\lambda_{0} \delta_{\alpha, \beta}$, and the matrix $K$ given by Eq. (8). The simultaneous invertibility of $\tilde{h}_{00}$ and $K$, namely,

$$
(\operatorname{det} K)\left(\operatorname{det} \tilde{h}_{00}\right) \neq 0
$$

guarantees the existence and uniqueness of $\rho^{(1)}$ and $\rho^{(2)}, \rho^{(0)}$ being fixed by the targeting condition (5). We checked that no further restrictions arise in the higher orders of the $1 / \Gamma$ expansion. Thus, the condition (A40), together with the main condition (5) form a complete set of sufficient conditions (apart from NESS uniqueness) for the NESS to approach the pure target state in the Zeno limit.

Finally, to estimate the convergence of $\rho_{\mathrm{NESS}}(\Gamma)$ to the pure target state, we investigate the purity $1-\operatorname{tr} \rho_{\mathrm{NESS}}^{2}(\Gamma)=$ 
$\Gamma_{\mathrm{ch}}^{2} / \Gamma^{2}+O\left(\Gamma^{-3}\right)$. A somewhat lengthy but straightforward calculation yields

$$
\Gamma_{\mathrm{ch}}^{2}=-2 \sum_{\alpha=1}^{d_{0}-1}\left|M_{0 \alpha}^{(1)}\right|^{2}-2 M_{00}^{(2)}
$$

From Eq. (A35), using $M_{00}^{(2)}=-\sum_{\alpha>0} M_{\alpha \alpha}^{(2)}$, we readily find $\Gamma_{\mathrm{ch}}{ }^{2}=\sum_{\alpha>0}\left(M_{V}^{(2)}\right)_{\alpha \alpha}=\sum_{\alpha>0} q_{\alpha}$, where $q_{\alpha}$ are determined by Eq. (A39). Multiplying (A39) by $K^{-1}$ and using (A22), we obtain Eq. (7).

Some remarks are in order about the invertibility of the matrix $K$. According to Eq. (8), $K$ is a real matrix. It has nonnegative off-diagonal elements and its diagonal el- ements satisfy $K_{\alpha \alpha}+\sum_{\beta \neq \alpha} K_{\alpha \beta}=-d_{\alpha}$, where $d_{\alpha}=$ $\sum_{k>0}\left|\left\langle 0\left|h_{k 0}\right| \alpha\right\rangle\right|^{2}$. By the Gershgorin circle theorem, every eigenvalue of $K$ lies within at least one of the disks on the complex plane centered at $K_{\alpha \alpha}$ and with radius $\sum_{\alpha \neq \beta} K_{\alpha \beta}$. All the disks lie completely in the negative half-plane, and some of them (those for which $d_{\alpha}=0$ ) touch the origin. If $d_{\alpha}>0$ for all $\alpha>0$, all the eigenvalues of $K$ are strictly negative and $K$ is invertible. If $d_{\alpha}=0$ for any $\alpha>0$, then $K$ becomes a stochastic matrix and has an eigenvalue 0 , i.e., it is not invertible. If only some $d_{\alpha}=0$, then, by the Gershgorin theorem, an eigenvalue 0 is not excluded. Calculating det $K$ via the Leibnitz formula, we find that for $\operatorname{det} K=0$, all Leibnitz terms must vanish separately. This allows, in principle, for a complete classification of the non invertibility points of $K$.
[1] S. Shankar, M. Hatridge, Z. Leghtas, K. M. Sliwa, A. Narla, U. Vool, S. M. Girvin, L. Frunzio, M. Mirrahimi, and M. H. Devoret, "Autonomously stabilized entanglement between two superconducting quantum bits," Nature 504, 419-422 (2013), letter.

[2] Y. Lin, J. P. Gaebler, F. Reiter, T. R. Tan, R. Bowler, A. S. Sorensen, D. Leibfried, and D. J. Wineland, "Dissipative production of a maximally entangled steady state of two quantum bits," Nature 504, 415-418 (2013), letter.

[3] H. Häffner, C. F. Roos, and R. Blatt, "Quantum computing with trapped ions," Physics Reports 469, 155 - 203 (2008).

[4] P. Schindler, M. Muller, D. Nigg, J. T. Barreiro, E. A. Martinez, M. Hennrich, T. Monz, S. Diehl, P. Zoller, and R. Blatt, "Quantum simulation of dynamical maps with trapped ions," Nat Phys 9, 361-367 (2013), article.

[5] D. Kienzler, H.-Y. Lo, B. Keitch, L. de Clercq, F. Leupold, F. Lindenfelser, M. Marinelli, V. Negnevitsky, and J. P. Home, "Quantum harmonic oscillator state synthesis by reservoir engineering," Science 347, 53-56 (2015).

[6] M. J. Kastoryano, F. Reiter, and A. S. Sørensen, "Dissipative preparation of entanglement in optical cavities," Phys. Rev. Lett. 106, 090502 (2011).

[7] F. Ticozzi and L. Viola, "Stabilizing entangled states with quasi-local quantum dynamical semigroups," Philosophical Transactions of the Royal Society of London A: Mathematical, Physical and Engineering Sciences 370, 5259-5269 (2012).

[8] Y. Ikeda and N. Yamamoto, "Deterministic generation of gaussian pure states in a quasilocal dissipative system," Phys. Rev. A 87, 033802 (2013).

[9] H. Krauter, C. A. Muschik, K. Jensen, W. Wasilewski, J. M. Petersen, J. I. Cirac, and E. S. Polzik, "Entanglement generated by dissipation and steady state entanglement of two macroscopic objects," Phys. Rev. Lett. 107, 080503 (2011).

[10] S. Diehl, E. Rico, M. A. Baranov, and P. Zoller, "Topology by dissipation in atomic quantum wires," Nat Phys 7, 971-977 (2011).

[11] J. Cho, S. Bose, and M. S. Kim, "Optical pumping into manybody entanglement," Phys. Rev. Lett. 106, 020504 (2011).

[12] E. G. Dalla Torre, J. Otterbach, E. Demler, V. Vuletic, and M. D. Lukin, "Dissipative preparation of spin squeezed atomic ensembles in a steady state," Phys. Rev. Lett. 110, 120402 (2013).

[13] Y.-D. Wang and A. A. Clerk, "Reservoir-engineered entangle- ment in optomechanical systems,” Phys. Rev. Lett. 110, 253601 (2013).

[14] F. Reiter, D. Reeb, and A. S. Sørensen, "Scalable dissipative preparation of many-body entanglement," Phys. Rev. Lett. 117, 040501 (2016).

[15] K. Stannigel, P. Rabl, and P. Zoller, "Driven-dissipative preparation of entangled states in cascaded quantum-optical networks," New Journal of Physics 14, 063014 (2012).

[16] F. Reiter, M. J. Kastoryano, and Sørensen A. S., "Driving two atoms in an optical cavity into an entangled steady state using engineered decay," New Journal of Physics 14, 053022 (2012).

[17] M. J. A. Schuetz, E. M. Kessler, L. M. K. Vandersypen, J. I. Cirac, and G. Giedke, "Steady-state entanglement in the nuclear spin dynamics of a double quantum dot," Phys. Rev. Lett. 111, 246802 (2013).

[18] Z. Leghtas, U. Vool, S. Shankar, M. Hatridge, S. M. Girvin, M. H. Devoret, and M. Mirrahimi, "Stabilizing a bell state of two superconducting qubits by dissipation engineering," Phys. Rev. A 88, 023849 (2013).

[19] J. T. Barreiro, M. Muller, P. Schindler, D. Nigg, T. Monz, M. Chwalla, M. Hennrich, C. F. Roos, P. Zoller, and R. Blatt, "An open-system quantum simulator with trapped ions," Nature 470, 486-491 (2011).

[20] G. Dirr, U. Helmke, I. Kurniawan, and T. Schulte-Herbrüggen, "Lie-semigroup structures for reachability and control of open quantum systems: Kossakowski-Lindblad generators form Lie wedge to Markovian channels," Reports on Mathematical Physics 64, 93 - 121 (2009).

[21] S. J. Glaser, U. Boscain, T. Calarco, C. P. Koch, W. Köckenberger, R. Kosloff, I. Kuprov, B. Luy, S. Schirmer, T. SchulteHerbrüggen, D. Sugny, and F. K. Wilhelm, "Training Schrödinger's cat: quantum optimal control," The European Physical Journal D 69, 279 (2015).

[22] G. Lindblad, "On the generators of quantum dynamical semigroups," Communications in Mathematical Physics 48, 119130 (1976).

[23] V. Gorini, A. Kossakowski, and E. C. G. Sudarshan, "Completely positive dynamical semigroups of $N$-level systems," Journal of Mathematical Physics 17, 821-825 (1976).

[24] N. Yamamoto, "Parametrization of the feedback hamiltonian realizing a pure steady state,” Phys. Rev. A 72, 024104 (2005).

[25] B. Kraus, H. P. Büchler, S. Diehl, A. Kantian, A. Micheli, and P. Zoller, "Preparation of entangled states by quantum markov 
processes," Phys. Rev. A 78, 042307 (2008).

[26] M. C. Fischer, B. Gutiérrez-Medina, and M. G. Raizen, "Observation of the quantum zeno and anti-zeno effects in an unstable system," Phys. Rev. Lett. 87, 040402 (2001).

[27] N. Syassen, D. M. Bauer, M. Lettner, T. Volz, D. Dietze, J. J. García-Ripoll, J. I. Cirac, G. Rempe, and S. Dürr, "Strong dissipation inhibits losses and induces correlations in cold molecular gases," Science 320, 1329-1331 (2008).

[28] F. Schäfer, I. Herrera, S. Cherukattil, C. Lovecchio, F. S. Cataliotti, F. Caruso, and A. Smerzi, "Experimental realization of quantum zeno dynamics," Nat Commun 5 (2014), article.

[29] A. Signoles, A. Facon, D. Grosso, I. Dotsenko, S. Haroche, J.M. Raimond, M. Brune, and S. Gleyzes, "Confined quantum zeno dynamics of a watched atomic arrow," Nat Phys 10, 715719 (2014), letter.

[30] P. Facchi and S. Pascazio, "Quantum zeno dynamics: mathematical and physical aspects," Journal of Physics A: Mathematical and Theoretical 41, 493001 (2008).

[31] J. J. García-Ripoll, S. Dürr, N. Syassen, D. M. Bauer, M. Lettner, G. Rempe, and J. I. Cirac, "Dissipation-induced hard-core boson gas in an optical lattice," New Journal of Physics 11, 013053 (2009).

[32] S. Dürr, J. J. García-Ripoll, N. Syassen, D. M. Bauer, M. Lettner, J. I. Cirac, and G. Rempe, "Lieb-liniger model of a dissipation-induced tonks-girardeau gas," Phys. Rev. A 79, 023614 (2009).

[33] P. Zanardi and L. Campos Venuti, "Coherent quantum dynamics in steady-state manifolds of strongly dissipative systems," Phys. Rev. Lett. 113, 240406 (2014).

[34] P. Zanardi and L. Campos Venuti, "Geometry, robustness, and emerging unitarity in dissipation-projected dynamics," Phys. Rev. A 91, 052324 (2015).

[35] R. Toskovic, R. van den Berg, A. Spinelli, I. S. Eliens, B. van den Toorn, B. Bryant, J.-S. Caux, and A. F. Otte, "Atomic spin-chain realization of a model for quantum criticality," Nat Phys 12, 656-660 (2016), letter.

[36] H.-P. Breuer and F. Petruccione, The Theory of Open Quantum
Systems (Oxford University Press, 2002).

[37] M. B. Plenio and P. L. Knight, "The quantum-jump approach to dissipative dynamics in quantum optics," Rev. Mod. Phys. 70, 101-144 (1998).

[38] S. R. Clark, J. Prior, M. J. Hartmann, D. Jaksch, and M. B. Plenio, "Exact matrix product solutions in the heisenberg picture of an open quantum spin chain," New Journal of Physics 12, 025005 (2010).

[39] V. Popkov and M. Salerno, "Anomalous currents in a driven $X X Z$ chain with boundary twisting at weak coupling or weak driving," Journal of Statistical Mechanics: Theory and Experiment 2013, P02040 (2013).

[40] V. Popkov, "Alternation of sign of magnetization current in driven $X X Z$ chains with twisted xy boundary gradients," Journal of Statistical Mechanics: Theory and Experiment 2012, P12015 (2012).

[41] D. Karevski, V. Popkov, and G. M. Schütz, "Exact matrix product solution for the boundary-driven lindblad $X X Z$ chain," Phys. Rev. Lett. 110, 047201 (2013).

[42] V. Popkov, D. Karevski, and G. M. Schütz, "Driven isotropic heisenberg spin chain with arbitrary boundary twisting angle: Exact results," Phys. Rev. E 88, 062118 (2013).

[43] T. Prosen, "Exact nonequilibrium steady state of a strongly driven open XXZ chain,” Phys. Rev. Lett. 107, 137201 (2011).

[44] G. T. Landi, E. Novais, M. J. de Oliveira, and D. Karevski, "Flux rectification in the quantum $X X Z$ chain," Phys. Rev. E 90, 042142 (2014).

[45] V. Popkov and C. Presilla, "Obtaining pure steady states in nonequilibrium quantum systems with strong dissipative couplings," Phys. Rev. A 93, 022111 (2016).

[46] V. Popkov, J. Schmidt, and C. Presilla, "Spin-helix states in the $X X Z$ spin chain with strong dissipation," (2017), arXiv:1703.08233.

[47] M. Žnidarič, "Relaxation times of dissipative many-body quantum systems," Phys. Rev. E 92, 042143 (2015).

[48] M. Žnidarič, "Dissipative remote-state preparation in an interacting medium,” Phys. Rev. Lett. 116, 030403 (2016).

[49] A. F. Otte, private communication. 